\title{
P-1153 - WhatsApp: a tool for multidisciplinary management in the Centre of Comprehensive Care for the Patient with Diabetes (CAIPaDi)
}

Ovalle-Escalera CN, García-Ulloa AC, Hernández-Jiménez SC

Center of Comprehensive Care for the Patient with Diabetes (CAIPaDi).

National Institute of Medical Sciences and Nutrition Salvador Zubirán

\section{Background}

Communication is essential within every medical department, particularly in interdisciplinary care centers. Members require to be aware of the patient's performance in every other health intervention in order to achieve premium care and optimize therapeutic strategies in every area.

Most health care providers who use WhatsApp form "groups" within the app. This allows efficient and useful communication between the team members in an easier way.

\section{Objective}

- Identify the major topics discussed in the group from December 2014 to March 2017.

- Analyze the number of text messages sent by each area of CAIPaDi

\section{Methods}

We introduced WhatsApp in CAIPaDi in 2013 in order to improve collaboration and clinical decisions. Each team member downloaded the application to their smartphone.

We created a group on WhatsApp where every member could be aware of the real-time transmitted information in the form of photos and text. (Fig. 1)

We conducted a descriptive analysis of the text messages sent from december 2014 to march 2017, divided them by category and area.

\section{Results}

A total of 22,988 text messages were transmitted in our WhatsApp group between December 2014 to March 2017, messages containing pictures were excluded. A total of 62 texts $(0.26 \%)$ were about hypoglycemia events and $124(0.53 \%)$ regarding patients who got excluded from the protocol were sent.

119 messages $(0.51 \%)$ about selection of leaders for the peer support program were delivered.

A total of $198(0.86 \%)$ alerts on hypertensive patients were sent, 120 (60.6\%) from the nursing department, $46(23.23 \%)$ from the physical therapy area and $32(16.16 \%)$ from endocrinology.

\begin{tabular}{|c|c|}
\hline Department & Number of text messages (\%) \\
\hline Psychiatry & $592(2.57 \%)$ \\
\hline Psichology & $510(2.21 \%)$ \\
\hline Nutrition & $472(2.05 \%)$ \\
\hline Endocrinology & $458(1.99 \%)$ \\
\hline Diabetes education & $284(1.23 \%)$ \\
\hline Physical therapy & $207(0.09 \%)$ \\
\hline Odontology & $198(0.86 \%)$ \\
\hline Nursing & $153(0.66 \%)$ \\
\hline Foot care & $152(0.66 \%)$ \\
\hline Ophthalmology & $124(0.53 \%)$ \\
\hline
\end{tabular}

Table 1. Number of messages sent by each department in CAIPaDi

\begin{tabular}{|cc|}
\hline Category & Number of text messages (\%) \\
\hline $\begin{array}{c}\text { Acknowledgement to } \\
\text { notifications }\end{array}$ & $11649(50.67 \%)$ \\
\hline Congratulations & $1919(8.34 \%)$ \\
\hline Answers & $1232(5.35 \%)$ \\
\hline $\begin{array}{c}\text { Messages from team } \\
\text { coordinators }\end{array}$ & $1097(4.77 \%)$ \\
\hline Metabolic alerts & $1000(4.35 \%)$ \\
\hline Administrative issues & $830(3.61 \%)$ \\
\hline Questions & $709(3.08 \%)$ \\
\hline Motivational & $369(1.6 \%)$ \\
\hline Thank you & $281(1.22 \%)$ \\
\hline Out of context & $238(1.03 \%)$ \\
\hline
\end{tabular}

Table 2. Categories of messages sent in the WhatsApp group

\begin{tabular}{l} 
Nursing \\
Good morning, the patient \\
AMCD reported 2 episodes \\
of hypoglycemia. The first \\
one was asymptomatic, \\
with a capillary glucose of \\
$44 \mathrm{mg} / \mathrm{dl}$ due to fasting. \\
The second one with \\
$33 \mathrm{mg} / \mathrm{dl}$ of glucose, no \\
symptoms \\
Diabetes education \\
OK, we will insist in the \\
importance of avoiding \\
fasting for more than 6 \\
hours \\
Psychiatry \\
She also commented about \\
an additional episode of \\
hypoglycemia a day before, \\
with 33 mg/dl of capillary \\
glucose, with diaphoresis \\
\hline
\end{tabular}

Figure 1. Example of a situation posed on the conversation

\section{Discussion}

The most frequent type of message sent was acknowledgement to notifications, which relates to a high response rate and awareness of messages between the team.

Despite the importance of all areas in CAIPaDi, psychology and psychiatry are two of the disciplines whose observations have the most impact on patient's assessment by other areas, these were disciplines that sent the most messages, mainly recommendations on patients' management for the rest of the areas.

We have observed a low rate of hypoglycemia and hypertensive crisis events in CAIPaDi, only $0.26 \%$ and $0.86 \%$ of the texts were related to these, constant communication contributed to their immediate treatment, which occurred in all cases.

\section{Conclusions}

The use of WhatsApp enhances communication between the health care providers belonging to a multidisciplinary team in a preventive health care setting such as CAIPaDi. The results of our study demonstrate that communication between the multidisciplinary team is adequate. The lack of cost, wide internet availability and the display of a notification when messages have been seen and received makes this application more convenient than calls or SMS. Thus, we strongly recommend its use.
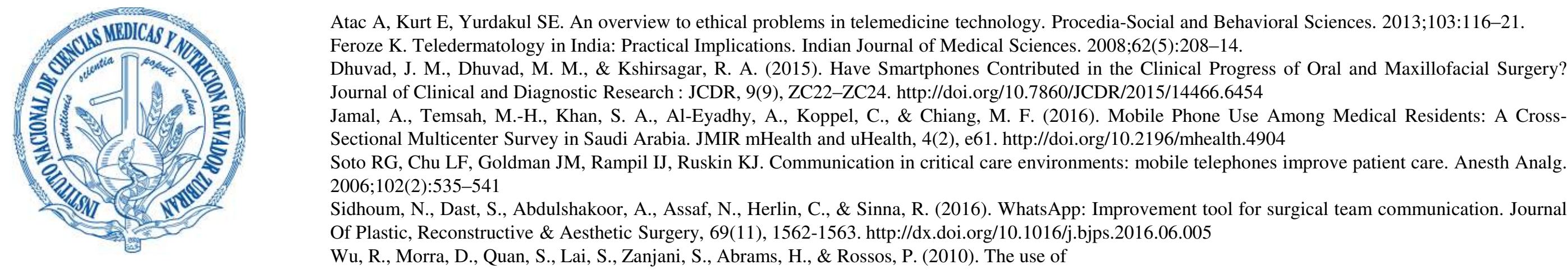

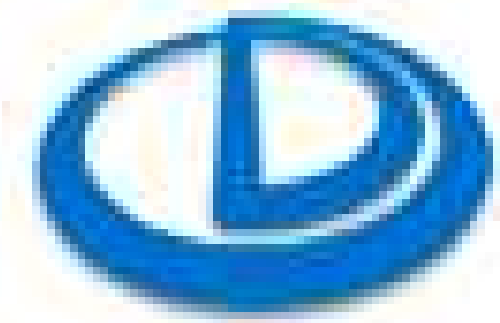

CAIPaDi 